\section{Mortalidade de mulheres em idade fértil devido a causas externas no Município de Cascavel, Paraná, Brasil, 1991 a 2000}

\author{
Mortality from injuries among childbearing-age \\ women in Cascavel, Paraná, Brazil, 1991-2000
}

Marcelo Pontual Cardoso 1

Aníbal Faúndes 2,3

\footnotetext{
1 Universidade Estadual do Oeste do Estado do Paraná Cascavel, Brasil.

2 Centro de Pesquisas em Saúde Reprodutiva de

Campinas, Campinas, Brasil. 3 Faculdade de Ciências Médicas, Universidade Estadual de Campinas, Campinas, Brasil.

Correspondência A. Faúndes Centro de Pesquisas em Saúde Reprodutiva de Campinas. C. P. 6181, Campinas, $S P$ 13.084-971, Brasil. afaundes@unicamp.br
}

\begin{abstract}
Injuries comprise the main group of causes of mortality among women 15 to 35 years of age in Brazil. The present study aimed to improve the knowledge concerning mortality by injuries, using a descriptive population-based approach, including all 869 death certificates for resident 10-49-year-old women from Cascavel, Paraná State, who died between 1991 and 2000. Mortality from injuries was analyzed according to cause, age, and calendar year. Pearson chi-square was applied at $p<0.05$. Injuries were the main group of causes of death up to 34 years of age and for all women 15-45 (coefficient 29.4/100,000 women) and the third most common among women 35 45. Traffic accidents were the first external cause of death in all age groups. We conclude that female mortality in Cascavel can be greatly reduced by interventions addressed to control external causes of death among young women.
\end{abstract}

Women; External Causes; Cause of Death; Mortality

\section{Introdução}

Nas últimas décadas surgiu uma justa preocupação com os níveis de mortalidade materna, e várias iniciativas foram tomadas com o intuito de combater as altas taxas de morte na gravidez, parto e pós-parto no mundo. Sem desconsiderar a enorme importância das mortes maternas, em sua maioria previsíveis, a pesquisa sobre elas, a partir da análise de todas as mortes de mulheres em idade fértil (10 a 49 anos de idade), levou a prestar atenção em outras causas de morte entre mulheres dessa faixa etária, que, de fato, representam um número muito maior de mortes, e também são essencialmente evitáveis.

Entre essas outras causas de morte de mulheres em idade reprodutiva, destacam-se as causas externas, que no Brasil constituem-se na primeira causa de morte de mulheres de 15 até os 35 anos, e ocupam o terceiro ou até o segundo lugar entre as causas de morte no grupo de 15 a 49 anos 1,2,3,4. O mais habitual é que as mortes em conseqüência de acidentes de tráfego ocupem o primeiro lugar nesse grupo de causas, seguidas pelos homicídios e suicídios 2,3,4.

Aparentemente, o predomínio das causas externas sobre outras causas é um fenômeno que se acentuou nos últimos anos. Um estudo realizado por Mello-Jorge et al. 5, em todo o Brasil, no período de 1977 a 1994, encontrou grande aumento da contribuição das causas externas para a mortalidade geral, havendo quase 
uma duplicação dos coeficientes gerais de mortalidade por essas causas entre o primeiro e o último ano do estudo. Ademais, notou-se um deslocamento da mortalidade por causas externas de 1977 a 1994 para faixas etárias mais jovens 5 . A mesma tendência foi observada nas sete principais capitais estaduais, no período de 1979 a 1998, principalmente entre pessoas jovens, de 15 a 24 anos de idade 6 . Em Campinas, São Paulo, comparando-se dois períodos (1985-1989 e 1990-1994) também se constatou aumento da mortalidade por causas violentas entre as mulheres em idade fértil, sobretudo devido aos homicídios 3 .

O Estado do Paraná tem-se destacado pelo bom funcionamento do Comitê Estadual de Mortalidade Materna, mas não há estudos mais detalhados sobre outras causas de morte. A região de Cascavel, de grande desenvolvimento recente da agroindústria, tem mostrado, nos últimos anos, taxas de mortalidade feminina em idade fértil superiores à média nacional e à do Estado do Paraná como um todo.

Considerando a importância crescente das causas externas de mortalidade no grupo de mulheres em idade reprodutiva, este estudo teve por objetivo analisar as mortes por causas externas no Município de Cascavel, em um período de dez anos (1991 a 2000) e, dentro desse grupo, avaliar as causas específicas de óbito mais freqüentes, incluindo a análise segundo grupos etários. Verificou-se, ainda, se houve mudanças nas razões de mortalidade proporcional do grupo de causas externas em relação ao número total de óbitos, comparando-se o primeiro com o segundo qüinqüênios estudados.

\section{Material e métodos}

O estudo incluiu inicialmente todos os óbitos declarados de mulheres de 10 a 49 anos de idade, residentes em Cascavel, ocorridos no período de 1o de janeiro de 1991 a 31 de dezembro de 2000. Foi identificado um total de 869 Declarações de Óbitos (DO), por todos os grupos de causas de óbito.

As DO foram selecionadas na Secretaria da Saúde do Município de Cascavel, em Cartórios de Registro de Óbito do Município e na Divisão de Dados de Mortalidade da Secretaria Estadual de Saúde, em Curitiba, e a seguir analisadas uma por vez, manualmente, quanto às variáveis consideradas para o estudo. Os dados constantes das DO foram então transcritos no formulário próprio do estudo.

As causas básicas do óbito foram codificadas de acordo com a Classificação Internacio- nal de Doenças, 10ạ Revisão (CID-10). Após codificação das causas básicas de óbito pela CID10 , as DO foram agrupadas de acordo com os grupos de causas básicas de óbito a que pertenciam, e então listadas. A seguir, as DO que tinham como grupo de causas básicas as causas externas (Capítulo XX da CID-10), foram separadas para continuidade da análise dos dados.

Os dados populacionais para o período entre os anos de 1991 a 2000 foram obtidos das estimativas anuais da população do sexo feminino, para o Município de Cascavel, por grupos etários (10-14 anos, 15-19, 20-24, 25-29, 30-34, 35-39, 40-44 e 45-49 anos), realizadas pelo Instituto Paranaense de Desenvolvimento Econômico e Social (IPARDES). A base de dados populacionais utilizada foi proveniente do X Censo Demográfico do Brasil, de 1991, e da Contagem Populacional, de 1996.

Foram identificados 206 casos de óbito (23,7\% do total) necessitando de investigação complementar. Esses casos foram selecionados quando na DO original não constava a causa básica do óbito, ou esta estava ilegível, quando no campo relativo à causa básica do óbito constava a declaração: "sem assistência médica" ou quando a causa básica declarada correspondia a um diagnóstico que parecia ser a causa terminal (e não a básica) do óbito. Nesse último caso, frente à duvida e não sendo possível estabelecer a cadeia de eventos que levou ao óbito, optou-se também pela investigação complementar.

Essa investigação constou de visita ao endereço da falecida, para as 206 ocorrências de óbito identificadas como necessitando de investigação complementar. Foi procurado então o parente mais próximo da falecida [marido, genitora, genitor, filha(o)], desde que maior de 18 anos. Nesta entrevista, foi realizado um questionamento livre acerca das condições de saúde durante a vida da falecida, com o objetivo de se resgatar a cadeia de eventos que levaram à morte, identificando-se então a causa básica do óbito. Em 108 vezes foi possível estabelecer a verdadeira causa básica do óbito por meio da entrevista com um parente da falecida.

Para 98 ocorrências de óbito, ou não foi encontrado nenhum parente da falecida no endereço (mudança da família) ou a entrevista não possibilitou a identificação da real causa básica do óbito. Em 28 casos, a identificação da causa básica foi realizada pela investigação nos prontuários em hospitais em que a falecida tenha sido atendida. Nos 70 casos restantes não foi possível estabelecer a causa básica do óbito, mesmo após a investigação complementar. Esses casos foram então classificados dentro 
do Capítulo XVIII (Causas Mal Definidas e Desconhecidas de Mortalidade) da CID-10.

Nenhum dos óbitos por causas externas necessitou de investigação complementar, visto que estavam todas as DO correspondentes bem preenchidas. Ademais, quando a investigação complementar foi realizada, não foi identificado nenhum caso de óbito relacionado à causa externa de mortalidade.

Após serem considerados completos os formulários próprios do estudo, por meio da revisão manual dos mesmos, os dados foram incluídos em um banco de dados em Epi Info 6.04 (Centers for Disease Control and Prevention, Atlanta, Estados Unidos), e realizada a análise estatística. As causas corrigidas de óbito foram agrupadas quanto aos capítulos da CID-10 e foram identificados os óbitos dentro do grupo Causas Externas de Mortalidade - Capítulo XX da CID-10 que foram detalhados quanto às suas causas específicas de óbito.

Posteriormente, as causas de óbito agrupadas foram listadas por qüinqüênio de idade das mulheres (10-14; 15-19; 20-24; 25-29; 30-34; 3539; 40-44; 45-49 anos), permitindo o conhecimento da distribuição percentual e dos coeficientes de mortalidade do grupo das causas externas por grupos etários.

As mortes por causas externas identificadas foram também divididas em dois grupos, de acordo com uma distribuição temporal: óbitos ocorridos no período entre 1991 e 1995 (1o qüinqüênio), e óbitos ocorridos entre 1996 e 2000 (2o qüinqüênio).

Foram calculadas as proporções do número de óbitos por causas externas em relação ao número total dos óbitos, obtendo-se a mortalidade proporcional por grupo de causas específicas causas externas, nos dois períodos de tempo considerados. A mortalidade proporcional deste grupo de causas foi comparada quanto aos dois qüinqüênios do estudo. Foi utilizado o teste do qui-quadrado de Pearson para verificar a independência da proporção dos principais grupos de causas específicas de óbito, nos dois qüinqüênios.

Para a avaliação das DO e prontuários de pessoas falecidas, foi solicitada autorização da autoridade competente e teve-se todo o cuidado necessário para preservar a confidencialidade da identificação de cada indivíduo. No caso das entrevistas domiciliares a familiares das falecidas, estas foram precedidas pela apresentação de um Termo de Consentimento Livre e Esclarecido (TCLE), sendo garantido o absoluto sigilo das pessoas entrevistadas. Esse TCLE foi aprovado pelo Comitê de Ética em Pesquisa da Faculdade de Ciências Médicas da Universidade Estadual de Campinas (FCM/UNICAMP).

\section{Resultados}

A soma da população de mulheres em idade fértil nos dez anos estudados foi de 745.404. O número de óbitos total nesse mesmo grupo e período foi de 869 , o que resultou num coeficiente de 116,6/ 100 mil mulheres em idade fértil para o período de dez anos do estudo, calculado por meio da somatória da população feminina em idade fértil, dividido pelo número total de óbitos femininos em idade fértil no período do estudo. Foi identificado o total de 219 óbitos por causas externas, o que significou que uma em cada quatro mortes de mulheres em idade fértil no período estudado foi devida a causas externas, resultando num coeficiente de mortalidade de 29,4/ 100 mil mulheres em idade fértil, o mais alto entre todos os grupos de causas básicas de óbito (Tabela 1).

\section{Distribuição dos casos de óbitos femininos por causas externas segundo local do óbito}

Menos da metade dos óbitos ocorreu em ambiente hospitalar $(46,1 \%)$, enquanto que $20,3 \%$ ocorreram no domicílio e mais de um quarto (25,8\%) na via pública (Tabela 2).

\section{Causas específicas de morte} entre as causas externas

Mais da metade das mortes por causas externas foram ocasionadas por acidentes de trânsito e quase um terço por homicídios e suicídios em proporções quase iguais. O restante foi causado por outros acidentes e causas variadas (Tabela 3).

Quanto à mortalidade por homicídios, a maior parte foi por arma de fogo (19 óbitos, contra 15 por arma branca e 3 por objeto contundente). Já os suicídios, estes foram realizados: por enforcamento, sufocação ou estrangulamento (11 óbitos) e por disparo de arma de fogo (10 óbitos) (dados não mostrados em tabelas).

\section{Mortalidade por causas externas segundo idade}

As causas externas ocuparam o primeiro lugar entre todos os grupos de causas de óbito nas mulheres mais jovens até o qüinqüênio de $30 \mathrm{a}$ 34 anos, e o terceiro entre as faixas etárias mais velhas, de 35 a 49 anos.

Os acidentes de trânsito constituíram-se na primeira causa de morte por causas externas em todos os grupos etários, mantendo-se com coeficientes entre 10 e 20 óbitos por $100 \mathrm{mil} \mathrm{mu-}$ 
Distribuição percentual e coeficiente de mortalidade das mulheres segundo grupos de causas (capítulos da CID-10) por 100 mil mulheres em idade fértil. Cascavel, Paraná, Brasil, 1991-2000.

\begin{tabular}{|c|c|c|c|}
\hline Grupos de causas & $\mathbf{n}$ & $\%$ & Coeficiente \\
\hline Causas externas de morbidade e de mortalidade (cap. XX) & 219 & 25,2 & 29,4 \\
\hline Doenças do aparelho circulatório (cap. IX) & 170 & 19,5 & 22,7 \\
\hline Neoplasias (cap. II) & 159 & 18,3 & 21,3 \\
\hline Doenças infecto-parasitárias (cap. I) & 43 & 4,9 & 5,8 \\
\hline Gravidez, parto e puerpério (cap. XV) & 41 & 4,7 & 5,5 \\
\hline Doenças do aparelho geniturinário (cap. XIV) & 37 & 4,3 & 5,0 \\
\hline Doenças do aparelho respiratório (cap. X) & 27 & 3,1 & 3,6 \\
\hline Doenças endócrinas (cap. IV) & 27 & 3,1 & 3,6 \\
\hline Doenças do aparelho digestivo (cap. XI) & 26 & 3,0 & 3,5 \\
\hline $\begin{array}{l}\text { Doenças do sangue e dos órgãos hematopoéticos (cap. III)/Transtornos } \\
\text { mentais (cap. V)/Doenças do sistema nervoso (cap. VI)/Doenças do sistema } \\
\text { osteomuscular (cap. XIII)/Lesões e envenenamentos (cap. XIX)/Fatores que } \\
\text { influenciam o estado de saúde e o contato com os serviços de saúde (cap. XXI) }\end{array}$ & 50 & 5,8 & 7,0 \\
\hline Mal definidas (cap. XVIII) & 70 & 8,1 & 9,4 \\
\hline Total & 869 & 100,0 & \\
\hline
\end{tabular}

Nota: população média estimada para o período $=74540,4$.

Tabela 2

Distribuição percentual dos óbitos por causas externas segundo local do óbito (capítulo XX da CID-10). Cascavel, Paraná, Brasil, 1991-2000.

\begin{tabular}{lrr}
\hline Local do óbito* & $\mathbf{n}$ & \multicolumn{1}{c}{$\%$} \\
\hline Hospital & 100 & 46,1 \\
Domicílio & 44 & 20,3 \\
Via pública & 56 & 25,8 \\
Outros & 17 & 7,8 \\
Total & 217 & 100,0
\end{tabular}

* Não houve informação sobre o local do óbito em dois casos.

lheres em cada grupo etário. As mortes por homicídio, suicídio e por outros acidentes apresentaram coeficientes semelhantes nas faixas etárias até o qüinqüênio de 30-34 anos, sempre em torno ou abaixo de 5 óbitos por $100 \mathrm{mil} \mathrm{mu-}$ lheres para cada uma dessas causas. Os homicídios tiveram um aparente aumento nos grupos de 35 a 39 e 40 a 44 anos, os suicídios nos grupos de 40 a 44 e 45 a 49 anos, e outros acidentes apenas no grupo de 45 a 49 anos (Figura 1).

\section{Mortalidade proporcional por causas externas segundo qüinqüênio}

Foi observada uma leve redução dos coeficientes de mortalidade por causas externas quanto ao qüinqüênio do estudo. Enquanto que para os anos de 1991 a 1995 foi anotado o coeficiente de mortalidade de 35,05/ 100 mil mulheres, para os anos de 1996 a 2000 este coeficiente foi de 24,42/100 mil. A diferença, entretanto, não foi considerada estatisticamente significante.

\section{Discussão}

O presente estudo mostrou que nos últimos dez anos do século XX o coeficiente de mortalidade feminina por 100 mil mulheres em idade fértil em Cascavel esteve ligeiramente acima do observado em outras cidades do país em anos anteriores. Enquanto que em Cascavel encontramos coeficiente de 116,6, Parpinelli 7 encontrou de 113,0 em Campinas entre 1985 e 1994, e Laurenti et al. 8, de 114,9 no Município de São Paulo em 1986.

Os três primeiros grupos de causas de óbito encontrados no presente estudo: causas externas, cardiovasculares e neoplasias aparecem também como os mais freqüentes em outras cidades, porém em ordem diferente. Enquanto em Cascavel o maior coeficiente foi observado nas causas externas, seguidas pelas doenças 
Distribuição percentual e coeficiente de mortalidade das mulheres segundo causas básicas de óbito do capítulo XX da CID-10 (causas externas) por 100 mil mulheres em idade fértil. Cascavel, Paraná, Brasil, 1991-2000.

\begin{tabular}{|c|c|c|c|}
\hline Causas básicas de óbito & $\mathrm{n}$ & $\%$ & Coeficiente \\
\hline \multicolumn{4}{|l|}{ Acidentes de trânsito $(n=115 ; 52,5 \%)$} \\
\hline $\begin{array}{l}\text { Ocupante de um automóvel traumatizado em um acidente de } \\
\text { transporte (V40-V49) }\end{array}$ & 74 & 33,8 & 9,9 \\
\hline Pedestre traumatizado em um acidente de transporte (V01-V09) & 39 & 17,8 & 5,2 \\
\hline Ciclista traumatizado em um acidente de transporte (V10-V19) & 2 & 0,9 & 0,3 \\
\hline \multicolumn{4}{|l|}{ Homicídios $(n=37 ; 16,9 \%)$} \\
\hline Agressões (X85-Y09) & 37 & 16,9 & 5,0 \\
\hline \multicolumn{4}{|l|}{ Suicídios ( $\mathrm{n}=32 ; 14,6 \%$ ) } \\
\hline Lesões autoprovocadas intencionalmente (X60-X84) & 32 & 14,6 & 4,3 \\
\hline \multicolumn{4}{|l|}{ Outros acidentes $(n=19 ; 8,7 \%)$} \\
\hline Afogamento e submersão acidentais (W65-W74) & 12 & 5,5 & 1,6 \\
\hline Quedas (W00-W19) & 7 & 3,2 & 0,9 \\
\hline \multicolumn{4}{|l|}{ Outras causas externas $(n=16 ; 7,3 \%)$} \\
\hline $\begin{array}{l}\text { Envenenamento [intoxicação] acidental por e exposição a substâncias } \\
\text { nocivas (X40-X49) }\end{array}$ & 5 & 2,3 & 0,7 \\
\hline $\begin{array}{l}\text { Acidentes ocorridos em pacientes durante a prestação de cuidados } \\
\text { médicos e cirúrgicos (Y60-Y69) }\end{array}$ & 5 & 2,3 & 0,7 \\
\hline Exposição à fumaça, ao fogo e às chamas (X00-X09) & 4 & 1,8 & 0,5 \\
\hline $\begin{array}{l}\text { Exposição à corrente elétrica, à radiação e às temperaturas e pressões } \\
\text { extremas do ambiente (W85-W99) }\end{array}$ & 2 & 0,9 & 0,3 \\
\hline Total & 219 & 100,0 & \\
\hline
\end{tabular}

Figura 1

Coeficiente de mortalidade de mulheres de 10 a 49 anos no período de 1991 a 2000 , por algumas causas externas por faixa etária.

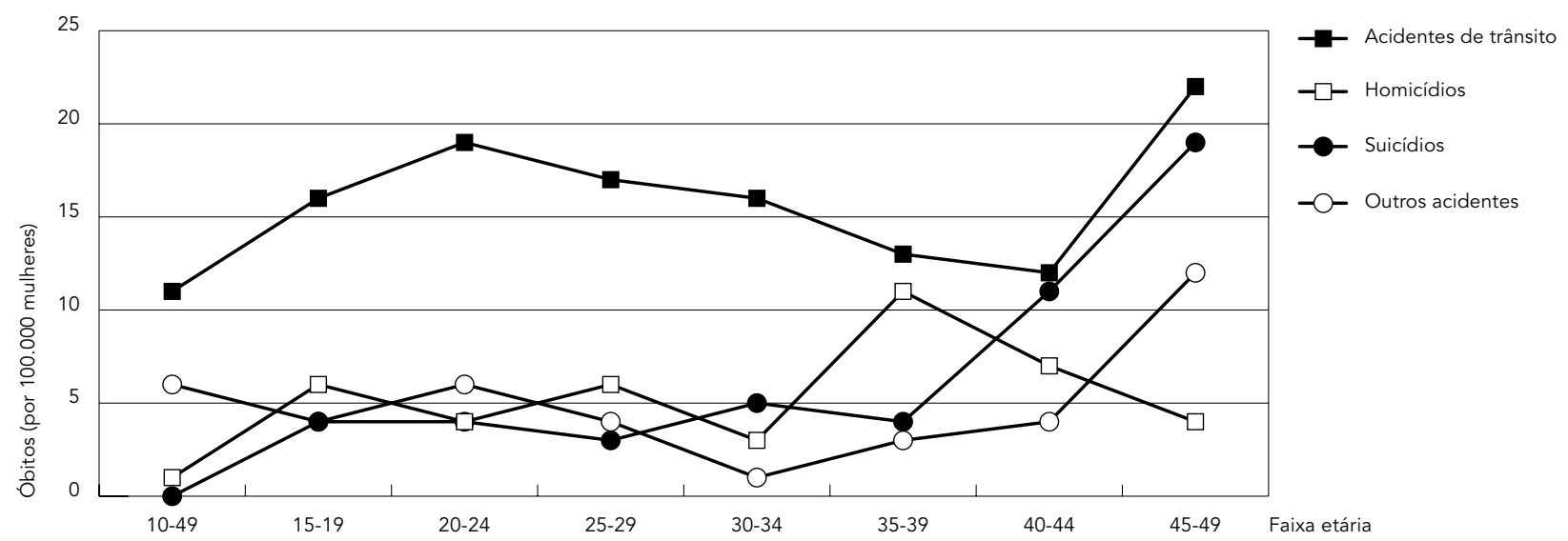


cardiovasculares e pelas neoplasias, e mais de longe pelas causas infecto-parasitárias e mortes maternas, em nenhuma das outras cidades estudadas as causas externas ocuparam o primeiro lugar. Apesar de diversos estudos terem mostrado a crescente participação das causas violentas na mortalidade, sobretudo nos grandes centros como a cidade do Rio de Janeiro 9, as causas externas foram a segunda causa de morte em Campinas 10 e em Brasília, Distrito Federal 4, de 1991 a 1995, e a terceira causa de morte de mulheres em idade fértil em Recife, Pernambuco 1, e em Ribeirão Preto, São Paulo 2, no período de 1985 a 1989. Estes dados sugerem que a maior mortalidade feminina em idade fértil observada em Cascavel está condicionada principalmente às mortes por causas externas. De fato, o coeficiente de mortalidade específico por causas externas foi de 29,4/ 100 mil mulheres em Cascavel comparados com 22,3 em Campinas 7 e 20,6 em São Paulo 8.

Analisando com mais detalhe as mortes por causas externas, verificamos que a diferença entre Cascavel e outras cidades parece estar influenciada pelos altos índices de mortes violentas relacionadas ao trânsito, visto que esta causa específica foi responsável por $51 \%$ das mortes por este grupo de causas em Cascavel, comparado com apenas $31,2 \%$ em Recife 1. Dentro do Estado do Paraná, Cascavel apresentou o segundo maior coeficiente de mortalidade por violência no trânsito, para o triênio 1999/ 2001 , sendo este coeficiente para ambos os sexos e todas as faixas etárias de 34,2/100 mil habitantes. Dentre todos os municípios paranaenses, apenas Cambé apresentou coeficiente maior, de 35,5/100 mil habitantes 11 .

É conhecida a importância dos acidentes de trânsito para a mortalidade no Brasil, que responderam por quase metade dos óbitos por causas externas em ambos os sexos em grande parcela das capitais brasileiras, com coeficientes de mortalidade quase sempre superiores a 20/100 mil habitantes, cifra muito mais elevada que a observada em outros países 12 .

Apesar de em outros estudos as causas externas não ocuparem o primeiro lugar entre as mortes femininas no conjunto de mulheres em idade fértil, em quase todos eles observou-se, como em Cascavel, que o grupo das causas externas ocupou sim o primeiro lugar como causas básicas de óbito nas faixas etárias mais jovens até os 34 anos de idade 4,10,13. Apenas em Recife as causas externas de mortalidade já não foram as mais freqüentes a partir da faixa dos 30 a 34 anos 1.

Uma característica das mortes por causas externas é sua relativa estabilidade nas diferen- tes faixas etárias. Apenas o grupo de 10 a 14 anos parece estar menos exposto, tanto neste estudo como no realizado em Campinas 10 . O aparente aumento no grupo de 45 a 49 anos observado em Cascavel pode ser apenas por acaso, dado o relativamente pequeno número de óbitos. Entretanto, como as outras causas mais importantes de óbito (neoplasias e doenças cardiovasculares) são raras nas idades mais jovens, as causas externas predominam como porcentagem do total dos óbitos nessas idades.

A importância dos acidentes de trânsito dentre as causas básicas de óbito em Cascavel se confirma ao verificarmos que os coeficientes específicos de óbitos por esta causa são sempre muito superiores aos observados em Campinas para cada grupo de idade. Enquanto em Campinas esses coeficientes mantém-se abaixo de 10/100 mil mulheres em todas as faixas etárias, exceto entre 15 e 19 anos, em que é apenas superior a dez 10, em Cascavel esses coeficientes estão sempre acima de 10 e mais freqüentemente acima de 15 óbitos por $100 \mathrm{mil}$ mulheres em cada grupo de idade.

Mesmo em áreas urbanas do interior do Brasil, onde a expressão da violência é menor em relação aos grandes centros urbanos, as conseqüências dos acidentes de trânsito constituem um dos principais agravos à saúde. Muito embora com coeficientes muito menores, que atingiram o valor de 4,5 óbitos por $100 \mathrm{mil} \mathrm{mu-}$ lheres em idade fértil no ano de 1992, também na cidade de Maringá, Paraná, que tem muitas semelhanças com Cascavel, a mortalidade por acidentes de trânsito foi expressiva 14 .

As agressões ou homicídios não tiveram a importância relativa dentre as causas externas de óbito como observado em Recife, onde ocuparam o primeiro lugar, com quase um terço dos óbitos neste grupo de causas 1, contrastando com menos de $17 \%$ das mortes por causas externas em Cascavel, sugerindo que esta causa talvez seja menos freqüente no Sul e Sudeste que no Nordeste.

Quanto ao coeficiente segundo idade, mostrou uma curva muito semelhante à observada em Campinas, com coeficientes mínimos na faixa de 10 a 14 anos e pico no grupo de 35 a 39 anos de idade 10. Estes dados são semelhantes aos de todo o Brasil, em 1988, em que o coeficiente mais baixo de mortalidade por homicídios também se observou na faixa etária entre 10 e 14 anos, e os maiores coeficientes tenderam a se concentrar nas faixas etárias entre 20 e 39 anos, mas com pico na faixa etária entre 20 e 29 anos 15 .

Quanto às “lesões autoprovocadas intencionalmente", ou suicídios, o coeficiente de mor- 
talidade de 4,3/100 mil mulheres observado em Cascavel foi semelhante a outros estudos brasileiros e em termos mundiais, podendo ser classificado como baixo 16, e comparável ao coeficiente geral (ambos os sexos, todas as idades) anotado para o Brasil 17.

Uma outra particularidade neste estudo é a alta porcentagem de óbitos ocorridos no domicílio $(20,7 \%)$ e na via pública $(9,1 \%)$. Na cidade do Recife, a mortalidade em domicílio ocorreu em $20,6 \%$ das vezes, mas apenas em $10 \%$ em Campinas 1,10 . O elevado percentual de óbitos na via pública pode ser explicado, ao menos em parte, pelo fato de que mais da metade dos óbitos por causas externas, verificados em nossa pesquisa, tenham sido por acidentes de trânsito tão violentos que as vítimas não tenham sobrevivido para chegar até o hospital. Em Recife, onde a mortalidade por homicídio foi muito maior que em Cascavel, a mortalidade em via pública foi menos da metade do que foi encontrado no presente estudo 1 . A maior proporção de óbitos em ambiente hospitalar em Campinas provavelmente indique uma melhor qualidade da assistência à saúde em Campinas, comparativamente a Cascavel, ou a um menor porcentual de causas acidentais, sobretudo acidentes de trânsito.

O grande limitante deste tipo de estudo é que depende em grande parte de informações preenchidas por outras pessoas, cuja preparação e motivação para preenchimento correto são muito variáveis. Assim, o preenchimento incorreto das DO foi o principal obstáculo para a realização desta pesquisa. Mesmo após a investigação complementar de 206 (23,7\%) do total das 869 DO incluídas na pesquisa, em 70 casos $(8,06 \%$ do total das DO) não foi possível estabelecer a causa básica do óbito. Isto indica a necessidade de se ressaltar aos médicos a im- portância do cuidadoso preenchimento das DO, reafirmando seu grande valor epidemiológico pelos dados que contêm. Deve ser esclarecido que a DO tem de ser preenchida da maneira mais acurada possível, pois não pode ser vista apenas como um documento necessário para o sepultamento. Da mesma forma, verifica-se a urgência da criação de um Serviço de Verificação de Óbitos em Cascavel, uma vez que muitas das DO selecionadas para investigação complementar o foram por constar como causa do óbito a declaração "sem assistência médica".

Até agora, os estudo publicados sobre mortalidade feminina em idade fértil no Brasil foram realizados nas capitais ou em cidades de grande porte. Acreditamos que este é o primeiro estudo deste tipo realizado numa cidade de tamanho médio no interior de um estado. Nossos resultados mostram que o Município de Cascavel não escapa de elevado obituário por causas violentas, sobretudo acidentes, o que pode ser atribuído à transição epidemiológica para a violência, que é mais descrita como ocorrendo nos grandes centros urbanos 4 . Assim, a mortalidade feminina em Cascavel poderia ser bem menor com medidas que pudessem controlar melhor as causas externas de morte.

Os resultados deste estudo sugerem a necessidade de estender este tipo de pesquisa a cidades menores e ao meio rural, para ter um panorama mais completo da mortalidade feminina em idade fértil, que vem sendo estudada apenas nas grandes cidades do país. Além disso, mostra que seria recomendável o monitoramento permanente da evolução da mortalidade feminina do município, como neste estudo, para verificar o resultado de eventuais medidas que deveriam ser tomadas, ou ainda, da falta de ações oportunas.

\section{Resumo}

Causas externas constituem-se na principal causa de óbito em mulheres de 15 a 35 anos no Brasil. Com o objetivo de contribuir para o conhecimento sobre a mortalidade por causas externas, realizou-se o presente estudo. O estudo foi descritivo de base populacional, incluindo todas as 869 Declarações de Óbitos de mulheres com idade de 10 a 49 anos, residentes no Município de Cascavel, Paraná, ocorridos entre 1991 e 2000. A mortalidade por causas externas foi analisada segundo causas, idade e ano de ocorrência. Aplicou-se teste do qui-quadrado de Pearson com $p<0,05$. As causas externas foram o principal grupo de causas de óbi- to (coeficiente 29,4/100 mil mulheres de 15 a 49 anos), o mais freqüente até a idade dos 34 anos e o terceiro entre 35 e 49 anos. Os acidentes de trânsito constituíram-se na primeira causa de morte por causas externas em todos os grupos etários. Concluímos que no $\mathrm{Mu}$ nicípio de Cascavel a mortalidade feminina poderia ser reduzida com medidas que controlassem as causas externas de morte entre mulheres jovens.

Mulheres; Causas Externas; Causa de Morte; Mortalidade 


\section{Colaboradores}

Ambos os autores idealizaram o estudo e preparam o projeto de pesquisa, analisaram os dados e redigiram o trabalho final. M. P. Cardoso foi responsável pela coleta e digitação dos dados.

\section{Agradecimentos}

Os autores agradecem a Maria Helena de Souza pelas orientações na analise estatística dos dados e aos Drs. Guilherme Cecatti, Mary Ângela Parpinelli e Fernanda Zurita pelas observações à pesquisa.

\section{Referências}

1. Albuquerque RM, Cecatti JG, Hardy E, Faúndes A. Causas e fatores associados à mortalidade de mulheres em idade reprodutiva em Recife, Brasil. Cad Saúde Pública 1998; 14 Suppl 1:41-8.

2. Carvalheiro CDG, Manço ARX. Mortalidade feminina no período reprodutivo em localidade urbana da região sudeste do Brasil. Evolução nos últimos 20 anos. Rev Saúde Pública 1992; 26:239-45.

3. Parpinelli MA, Faúndes A, Cecatti JG, Pereira BG, Passini Júnior R, Amaral E. Análise da mortalidade evitável de mulheres em idade reprodutiva. Rev Bras Ginecol Obstet 2000; 22:579-84.

4. Lacerda MVG, Gomes RA, Matta IT, Tauil PL. Mortalidade de mulheres em idade fértil no Distrito Federal, com ênfase na mortalidade materna. Brasília Med 1998;35:7-13.

5. Mello-Jorge MHP, Gawryszewski VP, Latorre MRDO. I - Análise dos dados de mortalidade. Rev Saúde Pública 1997; 31 Suppl:5-25.

6. Souza ER, Minayo MC, Malaquias JV. Suicide among young people in selected Brazilian State capitals. Cad Saúde Pública 2002; 18:673-83.

7. Parpinelli MA. Mortalidade de mulheres em idade reprodutiva no Município de Campinas: análise de 1985 a 1994 [Tese de Doutorado]. Campinas: Faculdade de Ciências Médicas, Universidade Estadual de Campinas; 1997.

8. Laurenti R, Buchalla CM, Lolio CA, Santo AH, Mello-Jorge MHP. Mortalidade de mulheres em idade fértil no Município de São Paulo (Brasil), 1986. I. Metodologia e resultados gerais. Rev Saúde Pública 1990; 24:128-33.

9. Chor D, Duchiade MP, Jourdam AMF. Diferencial de mortalidade em homens e mulheres em localidade da região sudeste, Brasil, 1960, 1970 e 1980. Rev Saúde Pública 1992; 26:246-55.
10. Faúndes A, Parpinelli MA, Cecatti JG. Mortalidade de mulheres em idade fértil em Campinas, São Paulo (1985-1994). Cad Saúde Pública 2000; 16: 671-9.

11. Secretaria de Estado da Saúde do Paraná. Mortalidade e violência. Boletim Epidemiológico 2002; Ano V:3-7.

12. Mello-Jorge MH, Latorre MRDO. Acidentes de trânsito no Brasil: dados e tendências. Cad Saúde Pública 1994; 10:19-44.

13. Haddad N, Silva MB. Mortalidade feminina em idade reprodutiva no Estado de São Paulo, Brasil, 1991-1995: causas básicas de óbito e mortalidade materna. Rev Saúde Pública 2000; 34:64-70.

14. Scalassara MB, Souza RKT, Soares DFPP. Características da mortalidade por acidentes de trânsito em localidade da região Sul do Brasil. Rev Saúde Pública 1998; 32:125-32.

15. Souza ER. Homicídios no Brasil: o grande vilão da saúde pública na década de 80. Cad Saúde Pública 1994; 10:45-60.

16. Diekstra RF, Gulbinat W. The epidemiology of suicidal behavior: a review of three continents. World Health Stat Q 1993; 46:52-68.

17. Krug EG, Powell KE, Dahlberg IL. Firearm-related deaths in the United States and 35 other high and upper-middle-income countries. Int J Epidemiol 1998; 27:214-21.

Recebido em 11/Jul/2005

Versão final reapresentada em 16/Nov/2005 Aprovado em 02/Mar/2006 